\title{
Finding 1-Day Vulnerabilities in Trusted Applications using Selective Symbolic Execution
}

\author{
Marcel Busch and Kalle Dirsch \\ \{marcel.busch, kalle.dirsch\}@ fau.de \\ IT Security Infrastructures Lab \\ Department of Computer Science \\ Friedrich-Alexander University Erlangen-Nürnberg (FAU)
}

\begin{abstract}
Trusted Execution Environments (TEEs) constitute a major building block for modern mobile devices' security architectures. Yet, the analysis tools available to researchers seeking to examine these critical components are rudimentary compared to the vast range of sophisticated tools available for other execution contexts (i.e., Linux or Windows userland). We see the primary reason for the lack of tools is originating from the closed-source nature of TEEs. Specifically, the analysis of Trusted Applications (i.e., userland applications executed in a TEE) is of vital importance, since they account for the largest attack surface. However, hardware primitives (i.e., ARM TrustZone) prevent access to this high-privileged context and thwart any form of dynamic analysis.
\end{abstract}

In this paper, we present our approach to investigate 1-day vulnerabilities using selective symbolic execution of real-world Trusted Applications (TAs). Our system, SimTA, is based on angr and emulates the TA's execution environment. We build SimTA based on insights gained from manual static analysis of a commercially and widely deployed closed-source TEE by using an exploit on a physical device. In our evaluation, we elaborate on how SimTA facilitates the binary-diff-guided analysis by replicating the analysis of a known critical vulnerability. Additionally, we reveal two further issues, an authentication bypass and a heapbased buffer overflow, that have quietly been introduced by the vendor.

\section{INTRODUCTION}

In 2016, at an event called "GeekPwn", Stephens [22] presented a chain of exploits that ultimately led to an arbitrary code execution within the TEE of Huawei [25]. Using these exploits, he could unlock the targeted device using the fingerprint sensor with a finger of any person or even a nose. His privilege escalation into the TEE is connected to CVE2016-8764, which is an input validation vulnerability that an attacker can leverage to execute arbitrary shellcode within the TEE context.

A common way to investigate vulnerabilities similar to this is binary-diffing in combination with meticulous manual analysis. To extract the patch for the vulnerability in question, we refer to CVE-2016-8764's summary [19] and identify the latest affected version to compare it with its succeeding

Workshop on Binary Analysis Research (BAR) 2020

23 February 2020, San Diego, CA, USA

ISBN 1-891562-62-2

https://dx.doi.org/10.14722/bar.2020.23014

www.ndss-symposium.org version. One problem that can arise while extracting the patch is that not only the vulnerable sequence of instructions appears in the binary-diff, but many others. For example, new features could have been introduced, or compiler flags might have changed, resulting in irrelevant sequences. In this case, indicators such as additional code accessing attacker-provided input, could be used to identify relevant sequences. Unfortunately, it is not possible to use dynamic analysis inside of the TEE to investigate the patches handling attacker-controlled input, because access is usually locked down by vendors. After finding a vulnerability, an analyst needs many parameters from the address space to replicate the exploit. However, the layout of the address space (i.e., the location where code and data are mapped to), which is necessary for the replication, is not publicly disclosed.

In this work, we present our insights and techniques to face these challenges. We studied CVE-2016-8764 using manual analysis guided by binary-diffing and performed a dynamic analysis on the device, treating the TEE as a black-box. We were successful in replicating Stephens' exploit and gained insights into Huawei's TEE, Trusted Core (TC). Using this exploit, we acquired the address space layout of the targeted TA. Next, leveraging the runtime parameters observed from the device, we implemented an angr-based [21] prototype, SimTA, capable of emulating the execution environment. SimTA achieves a runtime behavior that is close to the normal execution of the TA on the device.

In addition to having an execution environment for the targeted TA, SimTA allows us to annotate the attacker-controlled input, thus, permitting us to filter patches dealing with attackercontrolled input from the binary-diff. Furthermore, we can even selectively introduce symbolic inputs to better understand the constraints introduced by a patch. As a result, we found a previously unknown 1-day heap-overflow vulnerability, an authentication bypass, and the already known type-confusion vulnerability underlying CVE-2016-8764. We elaborate on the analyses that led to these findings in our evaluation.

In summary, our contributions are the following:

- We share our insights for the interfaces, the abstraction layers, and the address space layout of one TA for the TC TEE. In order to get access to TEE internals and examine the runtime parameters of the TA, we implement and use an exploit for CVE-2016-8764 to collect the information from a real device. 


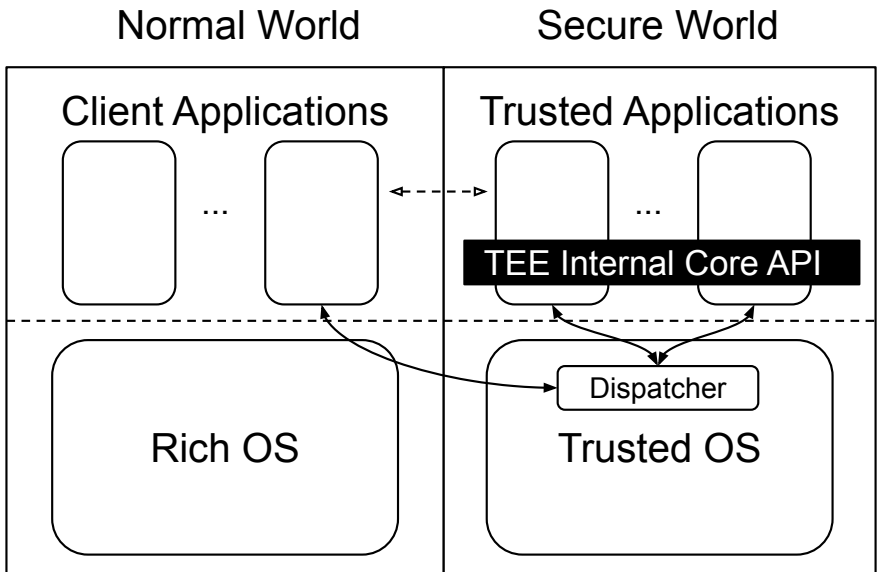

Fig. 1: Architecture and communication channels of modern TZ-based TEEs. The logical channel of a CA-TA interaction (dashed line) is carried out by both OSes that, cooperatively, forward and dispatch requests (solid lines).

- $\quad$ Based on these insights, we implement SimTA, an analysis tool capable of executing the target TA with selectively chosen symbolic inputs. We have opensourced SimTA to foster TEE-related research.

- We use SimTA to present a methodology to investigate binary-diff-guided analyses of security-critical patches. The effectiveness of our methodology is shown by replicating the analysis of CVE-2016-8764 and revealing two yet unknown critical issues affecting the same version.

Closed systems (i.e., TEEs) pose a significant obstacle for security research, because access is limited and information is scarce. With this research, we present an approach to enable the replication of CVEs and security fixes quietly introduced by vendors. By making our prototype publicly accessible, we hope to advance security research on TEEs. Our code and instructions on how to get the binaries used in our evaluation can be found on https:/github.com/teesec/simta

\section{BACKGROUND}

ARM TrustZone (TZ) [1] is commonly found on modern mobile devices. It allows partitioning of the System-on-Chip (SoC) into two execution contexts - the Secure World (SW) and the Normal World (NW) - where code and data from the SW cannot be accessed by the NW. The idea is to run a feature-rich operating system and its userland in the NW and only execute trusted code in the SW. Recent TZ-based TEEs split the SW into a kernel, the Trusted Operating System (Trusted OS), and a userland, which hosts TAs.

For data exchange, a logical communication channel exists between a CA and a TA (dashed line in Figure 1). Using this channel, CAs can request services from TAs. For example, requesting the generation of an asymmetric key pair, where the private key resides in the TEE, and the CA can use the public key [9]. In addition to the key generation, the TA would also provide an API to perform cryptographic operations using the safely stored private key (e.g., sign or decrypt messages).
Technically, the CA cannot call a TA directly. It needs to send its request to the Rich Operating System (Rich OS) that takes care of using a NW-SW shared memory region for the provided request data and initiates the world switch using a privileged instruction (e.g., smc). Then, the Trusted OS dispatches the request to the addressed TA. When the TA has processed the request, it writes its output to the shared memory region used for this session, returns to the Trusted OS, which, in turn, initiates the world switch back to the NW. Finally, the Rich OS returns execution to the CA. Figure 1 depicts this communication channel with solid lines.

Vendors of TEEs have an interest in providing a common interface for TAs in order to execute third-party TAs on their platforms. One set of standards that gains popularity in this regard is specified by GlobalPlatform (GP). GP is a nonprofit industry association that strives to enable collaborative and open ecosystems by developing specifications, especially regarding trusted computing technologies and particularly for TEEs. The specification relevant for our work is the GP TEE Internal Core API [7], which defines a common interface that can be used by TAs (depicted in Figure 1).

Studying vulnerabilities in TAs is especially important because TAs directly expose a large attack surface of the trusted computing base to the attacker-controlled NW. Our observation is that the number and complexity of pre-installed TAs are increasing. For example, in the case of the Huawei device we have in scope (i.e., the Huawei P9 Lite from 2016), we found 17 TAs. In contrast, we found 32 TAs on a recent model by Samsung (i.e., the Samsung Galaxy S9). SimTA's approach is a proposal to investigate security-critical bugs in TAs and, therefore, provides an option to learn from the mistakes made in the past.

\section{ChallengeS}

In this work, we are interested in dynamically analyzing patches introduced into TAs. In contrast to software executed in the NW, an analyst faces some challenges when trying to analyze TAs.

On modern Android devices, the trusted code (e.g., all code that runs in $\mathrm{SW}$ ) is proprietary, meaning there is no source code available. Furthermore, this code is executed in the SW, and, due to the TZ-provided isolation, we cannot make use of common tools utilized to study the runtime behavior of programs (e.g., debuggers). Also, we cannot modify a TA's code (e.g., binary instrumentation) because all code executed within the TEE is signed and gets verified before execution. Given these constraints, it seems unfeasible to perform advanced analyses to study patches of TAs on the device.

In order to study TAs during runtime, there are two apparent approaches. The first approach consists of taking the entire SW software stack in binary format (e.g., Trusted OS, and TAs) and execute it in a system emulator like QEMU. One of the problems with this approach is that SoCs used in production are usually not supported by full system emulators because their datasheets are not publicly available. An option to make this approach feasible is to reverse engineer the interaction between the Trusted OS and the hardware in order to implement a proper emulation. The interested reader may be referred to Harrison et al. [11] who studied this approach. 
The second approach focuses on the execution of individual TAs without the Trusted OS. This approach requires the knowledge of the virtual address space structure of the TA, the emulation of entries into and exits from the TA, as well as input and output mechanisms. For SimTA, we went with this approach and implemented a prototype for one of the TAs found on Huawei Android devices.

\section{RELATED WORK}

Vulnerability research on TEEs, so far, primarily happens using manual static analysis, and there are only a few cases known where researchers shared their insights.

Our work stands on the shoulders of Stephens' research [22], [23], who presented a chain of exploits targeting Huawei's TEE implementation, TC. Using this exploit chain, he could elevate his privileges from an Android application (the CA in this case) to the Trusted OS. From this work, particularly CVE-2016-8764 [19] is relevant for our research. Prior work on TC was carried out by Shen [20], who presented an escalation to the Trusted OS at BlackHat US 2015.

Further research targeting the Qualcomm Secure Execution Environment (QSEE) was conducted by Beniamini [3]-[6]. Beniamini, besides other issues, also elaborates on a chain of exploits leading to Trusted OS code execution. In addition, Quarkslab [10] conducted research on an OTP TA running on QSEE and shared their insights.

Besides TC and QSEE, Samsung devices used to ship with a TEE, which was first called MobiCore and later Kinibi. Atamali-Reihneh et al. [2] analyzed Samsung KNOX and its usage of the MobiCore TEE on a rather conceptual level. Later, Kinibi has been researched by Komaromy [13]-[15], and Beniamini [6] as well, where both analyses provide many details with technical depths.

As far as we know, all of the research mentioned above was carried out using manual static analysis and trial-and-error testing against a black-box on the phone as the only option for dynamic analysis. From our own work, we can tell that especially the last stage of exploit development (i.e., getting the exploit stable without the target crashing) targeting a TEE is laborious.

Recently, more attempts to emulate or re-host TEE components surfaced. For instance, CheckPoint Security [17] describe their work on a QEMU-based prototype capable of executing QSEE TAs. Their prototype forwards the syscalls of TAs to a manipulated TA inside of the TEE that acts as a proxy. The modifications of the proxy-TA are carried out using the flaws documented by Beniamini [4], [5]. Unfortunately, no source code of this prototype is publicly available yet.

An approach not requiring a physical device targeting TEEGris is pursued by Tarasikov [24]. TEEGris is a TEE implementation recently introduced on Samsung phones that presumably replaced Kinibi. Tarasikov is working on a QEMUusermode implementation to execute TEEGris TAs.

A re-hosting solution capable of performing full-system emulation of TEEs named PartEmu will be presented at Usenix 2020 by Harrison et al. [11]. TC, the TEE in scope for our research, is not part of PartEmu's evaluation.
Lastly, Hua et al. [12] proposed a system called $v T Z$ capable of virtualizing TEEs in ARM TZ. This work does not have the execution of closed source TEEs in scope, and extending its scope would require significant reverse-engineering effort in order to support the hardware requirements of different proprietary TEEs available on the market.

In our approach, we build on top of the gained vulnerability research and connect our insights with an angr-based approach to execute TAs for TC.

\section{UnDERSTANDING TRUSTED APPLICATIONS FOR TRUSTEDCORE}

In this section, we elaborate on the structure of TC-based TAs and share insights gained from an exploit on a physical device. The insights from this section will later allow us to implement SimTA, an angr-based prototype capable of emulating the execution environment of the target TA. We limit our scope to one TA (storageTA) that can be found on Huawei devices, although many insights apply generically to TC TAs (i.e., they all use GP standards and, presumably, the same program loader).

\section{A. TA Lifecycle}

Our first observation by looking at storageTA is that it makes use of the GP TEE Internal Core API specification [7]. This API defines the lifecycle of TAs. A simplified version of TC's implementation of this lifecycle is given in Listing 1 The MsgRcv() and MsgSend() functions represent the integration of the TA with the dispatcher (see Figure 11). The lifecycle functions have the following purpose:

- TA_createEntryPoint. Constructor, called once during initialization of TA.

- TA_OpenSessionEntryPoint. Opens client session, the provided session object is used to maintain state for the session.

- TA_InvokeCommandEntryPoint. Invocation of trusted application commands.

- TA_ClosesessionEntryPoint. Closes client session, frees space for the session object.

- TA_DestroyEntryPoint. Destructor, called once during tear down of TA.

The most interesting data structures processed by this API are passed to TA_OpensessionEntryPoint and TA_InvokeCommandEntryPoint. The arguments cm$\mathrm{dId}$, paramtypes, and params originate from a CA in the NW and, therefore, are user controlled.

\section{B. TA Commands}

Inside of the TA_InvokeCommandEntryPoint lifecycle call, the actual interface of the TA is exposed. As depicted in Listing 2 (a simplified version of storageTA), the TA-specific command is chosen via the cmdId argument. 


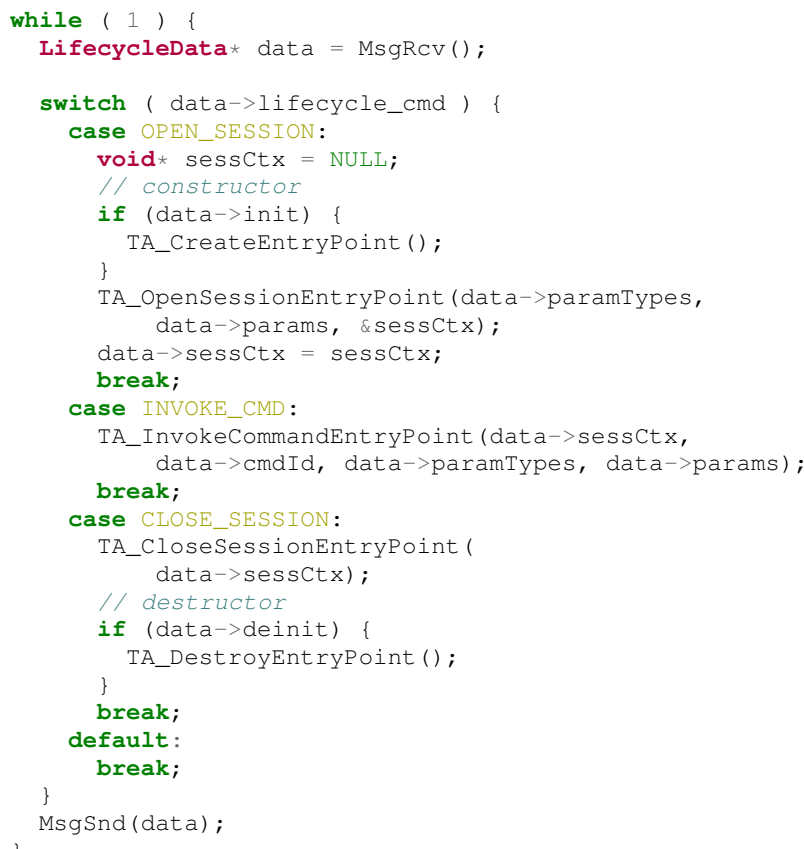

Listing 1: TC TAs follow the lifecycle specified in the GP Internal Core API.

From this listing, we can see that params is either a memory reference or a value. The corresponding union definition is given in Listing 3. The params argument of TA_InvokeCommandEntryPoint contains up to four pointers to a struct like this.

Now it also becomes clear that the paramTypes argument is necessary for the TA to get to know if the CA sent a buffer or values. For each command, the TA has to check the paramTypes to prevent wrong assumptions about how the provided data can be interpreted.

From Listing 2, we can also see that the TA itself can have a stateful API. The state for storageTA is stored within the sessCtx argument so that subsequent calls from the same CA can operate on this state (e.g., read from a previously opened file handle).

Looking at the functions used inside the different commands (e.g., TEE_OpenPersistentObject ()), we can see that most of them map to the GP Internal Core API [7]. Apparently, storageTA itself is just a thin layer of code that connects the TEE lifecycle with the storage API defined in the GP Internal Core API.

\section{TA Address Space Layout}

From the previous two sections, we can learn about the integration of the TA into the TEE (e.g., how the dispatcher provides data) and how the GP Internal Core API encapsulates most of the functions used by a TA. In order to emulate the execution environment on the device, however, we need the TA's address space layout. This layout will later be used as an input for SimTA.

To retrieve these runtime parameters, we build on the research of Stephens [22]. He found a type-confusion bug

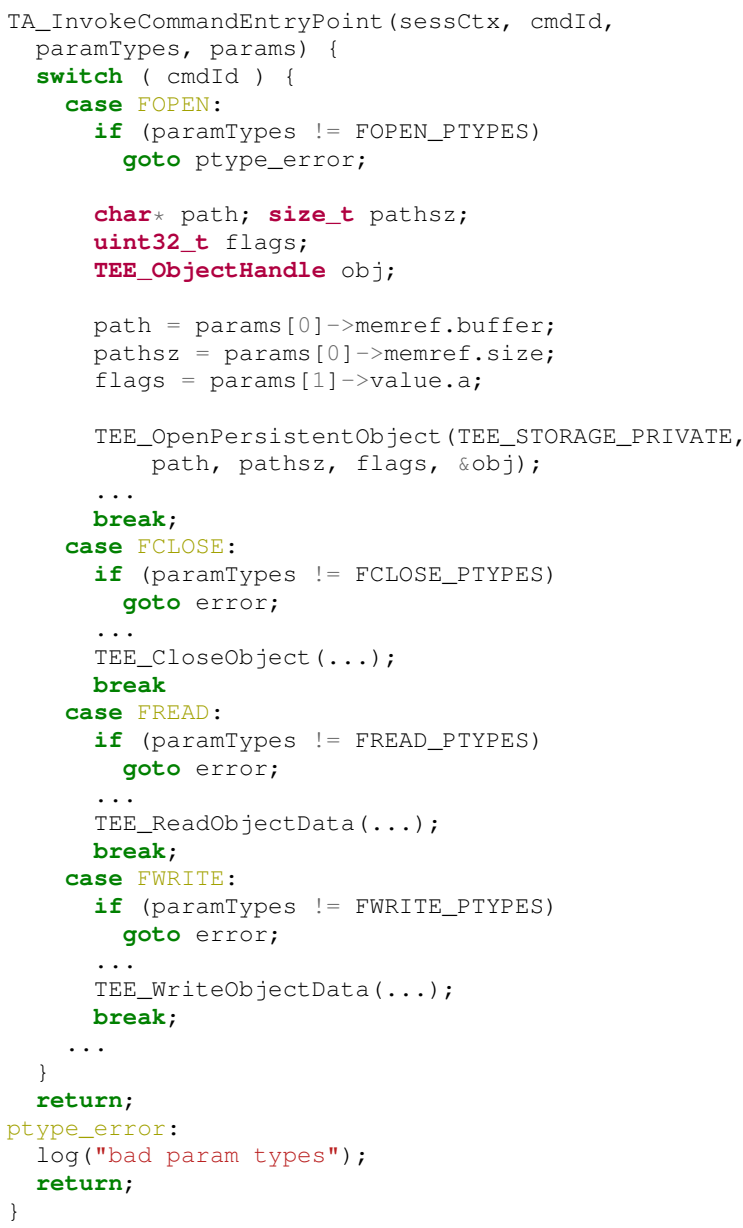

Listing 2: Each TC TA has a cmdId-handler that implements different commands. This is a simplified handler of storageTA.

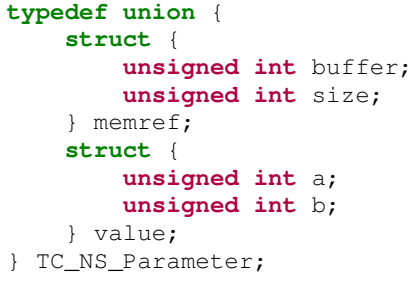

Listing 3: An array of four TC_NS_Parameter unions is the primary data structure for input and output for TC TAs.

in storageTA that he could use to gain code execution in this TA's execution context. Since no proof of concept was available for this exploit, we needed to replicate his research and develop the exploit ourselves. Stephen's slides were invaluable for this process. Using the exploit, we were able to leak arbitrary memory from storageTA's execution context and reconstruct its address space. The reconstructed address space is depicted in Figure 2

The first element mapped is globaltask which is a binary without ELF header that can also be extracted from the device's firmware image. Fortunately, we found a string table and a symbol table at the end of the binary. Using symbols like TEE_TEXT_START, TEE_BSS_START, and 


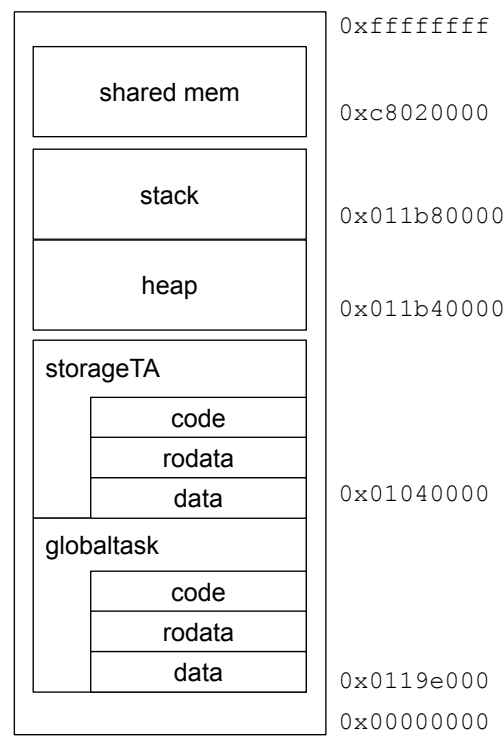

Fig. 2: Virtual address space of storageTA as extracted from a real device using an exploit based on CVE-2016-8764.

TEE_GOT_START, we were able to reconstruct globaltask's sections. globaltask's code and data sections are mapped according to the assigned virtual addresses from the ELF section headers. Directly above globaltask, we can find the storageTA binary itself. globaltask turns out to contain the implementation of all GP Internal Core API functions that are called by storageTA and it is the only mapped library. Consequently, globaltask should only be dependent on the syscalls of the Trusted OS. By leaking the content of storageTA's .bss segment that contained references to the heap, we were able to locate the heap. Using a leaked stack pointer ( $r 13$ ) we could also reveal the stack location. Additionally, a shared memory region is mapped into the address space which is used for data exchange between SW and NW.

\section{Generalizing SimTA}

The insights gathered regarding TC TAs are based on the static and dynamic analysis of one TA (e.g., storageTA). However, the firmware we investigated contains multiple TAs, namely 1 ,

$$
\begin{aligned}
& \text { - task_keyboard } \\
& \text { - task_storage } \\
& \text { - task_gate- } \\
& \text { keeper }
\end{aligned}
$$$$
\begin{array}{ll}
\text { - } & \text { task_keymas- } \\
& \text { ter } \\
\text { - } & \text { task_reet } \\
\text { - } & \text { task_secboot }
\end{array}
$$

By looking at these TAs, we could verify that all of them make use of the TA lifecycle and TA command processing, as described above. Since we do not have an exploit to study the address spaces of these TAs, we were not able to verify if our

\footnotetext{
${ }^{1}$ The names are taken from header fields within the firmware image. task_storage corresponds to storageTA.
}

gained insights from storageTA's address space also hold for these TAs. A reasonable assumption is, however, that the same loader loads all TAs.

Besides the TAs found in the firmware image, we identified encrypted TAs that reside in the Android filesystem (e.g., all /vendor/bin/*. sec files). Since we could not analyze these TAs statically nor dynamically, we cannot verify our gained insights for these TAs as well.

In order to get an idea of our insights' general applicability, we looked into OP-TEE [16] TAs. OP-TEE is an open-source reference implementation for ARM TZ-based TEEs maintained by Linaro. We found that OP-TEE TAs use the same lifecycle functions as TC (see Section V-A). One of the major differences is the lifecycle's integration with the dispatcher. Instead of the while(1)-loop structure communicating with the dispatcher using MsgRcv() and Msgisnd() (as described in Listing 1), OP-TEE uses two functions called utee_entry and utee_exit to enter and exit TAs. The cmdId-handler of OP-TEE TAs is similar to the one used by TC TAs. Apart from this, since OP-TEE's TA loader and libraries used by TA's are open source, it should be straightforward to adapt SimTA for OP-TEE TAs.

Other platforms potentially in scope for SimTA are Samsung's TEEGris, Trustonic's Kinibi, and Qualcomm's QSEE. An indicator of GP standards usage for these TEEs can be found in the publicly available list of GP members [8]. Besides Huawei, also Samsung, Trustonic, and Qualcomm are full members of GP and, according to GP's website, share a common goal of developing GP's specifications. Additionally, we could verify that concepts from the GP specification are being introduced into the Linux kernel for Qualcomm chipsets ${ }^{2}$

In summary, a broader technical study is necessary to evaluate if a system like SimTA can be extended to other TEEs used in production. But, it seems that the ecosystem is converging towards a common specification, which is a good sign for the reusability of SimTA's components.

\section{IMPLEMENTATION}

We were able to extract the storageTA ELF directly from the target device's firmware image. Since TC on the ARM-based target device runs in 32-bit mode, all TAs on this platform are 32-bit binaries too. In this version, TC does not make use of program headers and uses the virtual address of a section provided in the ELF file to load it into memory.

Our SimTA prototype is implemented based on angr version 8.19.4.5 and Python 3.6.7. As mentioned in Section V-A TAs follow a lifecycle that is usually implemented using a while(1)-loop which, at its start, receives data from the execution environment, and, at its end, returns data to the execution environment. Since we want to emulate this environment using SimTA, we hook the functions interacting with it using angr's SimProcedures. We chose the first instruction of the lifecycle-loop to be the entry point into the binary. Before execution begins, we initialize the state angr is working on using the address space parameters derived from our analysis described in Section $\mathrm{V}-\mathrm{C}$. This address space

\footnotetext{
${ }^{2}$ https://android.googlesource.com/kernel/msm/+/refs/heads/android-msmmarlin-3.18-pie/drivers/misc/qseecom.c
} 
information includes loading storageTA and globaltask as well as initializing the stack, the heap, and the shared memory region. Regarding registers, we observed that only two registers needed to be initialized with references to the current stack frame. These two references could easily be identified from the disassembly by looking at framepointer-relative address assignments to those registers.

Moreover, we implemented a subset of the GP TEE Internal Core API as angr SimProcedures. This subset is chosen in order to support the execution of storageTA. Additionally, we implemented a simple heap implementation to support TEE_Malloc and TEE_Free. From reading the GP Internal Core API, we know what a particular function is supposed to do, and for all functions used by storageTA, we were able to implement SimProcedures that keep the TA in a useful state for the binary-diff analysis.

SimTA allows us to pass data consumed by the lifecycle functions from the MsgRcv ()-function (Listing 1) into the storageTA process. This way, we can pass data into the process from the perspective of a CA. By setting input data to symbolic values, we are able to perform advanced analyses, as we will elaborate on in the next section.

\section{EvaluATION}

In this section, we evaluate SimTA in regard to its performance and effectiveness.

\section{A. Performance}

When executing a binary in an emulated environment, a performance impact is natural. Regarding SimTA, which is based on angr, the target TA's ARM instructions are first lifted to VEX IR (i.e., Valgrind's intermediate representation [18]) and then evaluated by a component called SimEngineVEX. To estimate the slow down of the emulation compared to the original execution environment (i.e., the device), we perform the following sequence of actions in both environments 100 times:

1) load the storageTA
2) open a session
3) create and open a secure file
4) write to the secure file
5) seek to the beginning of the secure file
6) read from the secure file
7) close the secure file
8) close the session
9) unload the storageTA

On average, the execution of this sequence on the device takes 36 milliseconds and 39202 milliseconds using SimTA, which results in a slow down factor of approximately 1089x. Note, that this significant slow down is a conservative measurement since we did not optimize SimTA for performance.

Overall, SimTA trades performance for control, but, from our experience, this performance impact is acceptable for the analyses conducted with SimTA.

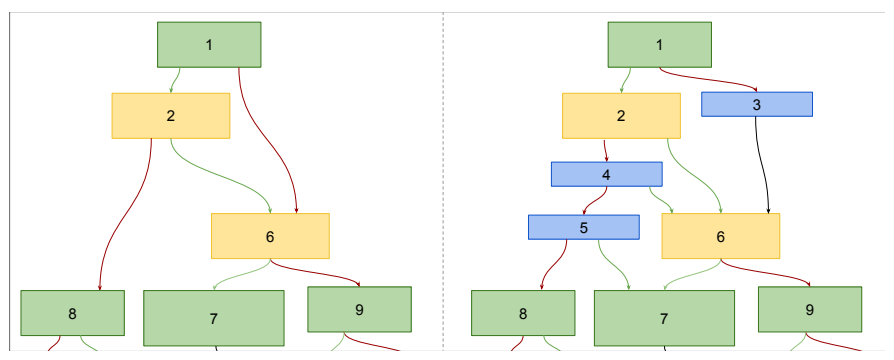

Fig. 3: This figure shows an extract of storageTA's control flow graph - unpatched version left and patched version right. The red, green, and black arrows indicate conditional and unconditional control flow transitions. Unchanged, changed, and new basic blocks are color-coded in green, yellow, and blue, respectively.

\section{B. Effectiveness}

In order to evaluate the effectiveness of SimTA, we generate a binary-diff of storageTA from firmware version VNSL21C432B130 to version VNS-L21C432B160 and analyze the diff. For ease of reference, we refer to the earlier version as sta and the later version as sta'.

First, we manually generate a binary-diff using Zynamic's BinDiff and filter for relevant patches. From BinDiff's "Matched functions" view, we can identify matched functions containing changes. Using the flow graphs feature, we can view a color-coded control flow graph of both versions of a matched function side-by-side, as depicted in Figure 3 As a heuristic to filter relevant patches, we concentrate on blue-colored basic blocks that access attacker-controlled input. These basic blocks represent new basic blocks in the patched version (e.g., sta'). In order to do the filtering, we manually extract the blue basic blocks from BinDiff and perform an analysis with SimTA on sta' reveiling if attacker-controlled input (i.e., paramTypes and params as passed to the lifecycle functions in Listing 1 ; is accessed. If no attacker-controlled input is accessed, we do not consider the change for further analysis.

Second, we run a further analysis using SimTA to examine the constraints introduced by the patches. From each filtered blue basic block (i.e., basic blocks 4 and 5 remain), we look for the next subsequent basic blocks that have equivalent basic blocks in sta (i.e., for basic block 5, these would be basic blocks 7 and 8). Since we know from the previous analysis which part of the attacker-controlled input is accessed in basic block 5, we can selectively set this input to a symbolic value, and execute both versions of storageTA to a common basic block (e.g., basic block 8). Lastly, we can view and compare the constraints in both versions using SimTA, and reason about the security impact of the introduced patches.

In the following paragraphs, we elaborate on four analyses performed using SimTA.

One of the changed functions, strToByte (), is a hexdecode function and contains changes according to BinDiff. By passing the new basic blocks to SimTA, we can see that these basic blocks do not access attacker-provided input and, therefore, we exclude it from further analyses. 
A further function containing changes is the lifecycle function TA_InvokeCommandEntryPoint known from Section V-A Within the set of introduced basic blocks for this function, SimTA identifies basic blocks operating on the paramTypes argument. By selectively setting paramTypes symbolic and examining the constraints in both versions of storageTA, we unveil missing parameter type checks for all commands in this TA. This forgotten check is particularly critical for the FREAD and FWRITE commands, since these commands operate on attacker-provided buffers. If we can control the location of these buffers, we have an arbitrary read or write primitive. It is no surprise that we find a bug like this in storageTA since these are exactly the primitives Stephens [22] used in his work. Using SimTA, we rediscovered the type-confusion underlying CVE-2016-8764.

Another interesting finding revealed by SimTA is an introduced length check for a buffer in the FOPEN command. This 1-day bug was discovered because of an additional length check of a buffer contained in the params argument. SimTA enabled us to identify this check and investigate the consequences of its absence in sta. For this bug, it was necessary to investigate sta at a much later location than the actual check was introduced. Its severity becomes clear by looking at an unconstrained attacker-controlled size and attacker-controlled source buffer that gets passed into a memory copy function having a fixed-sized heap-based destination buffer. An attacker can corrupt the heap using this vulnerability and potentially gain code execution within sta. As should be clear from the context, this bug is fixed, but it is a perfect example of how SimTA supports binary-diff-guided analyses for TAs.

Our last discovery covers the authentication logic present in the TA_OpenSessionEntryPoint lifecycle function. Within this logic, an identifier of the calling CA and a constant client signature is checked. Aside from the fact that we can easily fake these inputs since they are controlled by the user, we can see diverging constraints using SimTA in this logic. There are two allowed identifiers, and from the introduced constraints, it becomes clear that the signature for the identifier / system/bin/tee_test_store is not checked at all in sta. Presumably, this identifier is used for testing purposes and should not be part of the code used in production. Using this identifier, we can get access to the core logic of sta without providing any client signature.

In summary, we provide evidence for the effectiveness of SimTA by re-discovering the type confusion bug underlying CVE-2016-8764 and identifying two further patches for critical issues in storageTA.

\section{LIMITATIONS}

SimTA was not developed, having a holistic emulation of the TEE in mind. It is rather an approach to have an effective and fast way to retrieve runtime information from two TAs one being a patched version of the other - and facilitate the analysis of introduced patches.

We are aware of two aspects of the TC TEE that we do not emulate correctly. In particular, we did not initialize and hook up the heap implementation that comes with globaltask. SimTA's heap implementation is basic and does not consider fragmentation of the heap or merging of heap chunks. Moreover, we do not implement the 8-bit entropy Address Space Layout Randomization (ASLR) used on the device [22].

We only implemented the SimProcedures from the GP TEE Internal Core API that are needed to execute storageTA. These SimProcedures are made publicly available and can be used as a starting point to support more functions of this API. In summary, we are confident that SimTA can be extended to other TC TAs or even other TEE implemenations that comply with GP specifications.

\section{OUTLOOK AND Future WORK}

Our future work will extend SimTA to more TAs. Besides TC, there are TEEGris and OP-TEE that are known to follow GP specifications. From recent Linux kernel sources for Qualcomm SoCs, we can also see that GP concepts have been introduced. Consequently, the approach chosen by SimTA might be viable for all major TEEs.

One arising problem in this area is that vendors start to lock down their systems. For instance, Huawei stopped providing bootloader unlock codes, which are necessary to customize the NW software stack in mid-2018. This situation leaves security researchers with interest in TEEs with many hoops to jump through before they can analyze their targets.

\section{Conclusions}

In this paper, we presented our ongoing research in finding 1-day vulnerabilities in TAs. We developed SimTA, an angrbased solution to emulate the TEE runtime for TAs found on Huawei devices. Using SimTA, we replicated a known bug in Huawei's storageTA and revealed two additional critical issues that have been silently patched by the vendor.

We hope to foster TEE-related security research with this work and provide our tools to reduce the effort to enter this interesting field of research for other security researchers.

\section{ACKNOWLEDGMENT}

This research was supported by the German Federal Ministry of Education and Research as part of the Software Campus project.

\section{REFERENCES}

[1] ARM, "Arm security technology: Building a secure system using trustzone technology," http://infocenter.arm.com/help/topic/com.arm. doc.prd29-genc-009492c/PRD29-GENC-009492C_trustzone_security_ whitepaper.pdf 2008, accessed: 2019-08-28.

[2] A. Atamli-Reineh, R. Borgaonkar, R. A. Balisane, G. Petracca, and A. Martin, "Analysis of trusted execution environment usage in samsung knox," in Proceedings of the 1st Workshop on System Software for Trusted Execution, 2016, pp. 1-6.

[3] G. Beniamini, "Cve-2015-6639 exploit," https://github.com/ laginimaineb/cve-2015-6639 2016, accessed: 2019-08-28.

[4] G. Beniamini, "Exploring qualcomm's secure execution environment," https://bits-please.blogspot.com/2016/04/exploring-qualcommssecure-execution.html 2016, accessed: 2019-08-28.

[5] G. Beniamini, "Qsee privilege escalation vulnerability and exploit (cve-2015-6639)," https://bits-please.blogspot.com/2016/05/qseeprivilege-escalation-vulnerability.html 2016, accessed: 2019-08-28. 
[6] G. Beniamini, "Trust issues: Exploiting trustzone tees," https://googleprojectzero.blogspot.com/2017/07/trust-issues-exploitingtrustzone-tees.html 2017, accessed: 2019-08-28.

[7] GlobalPlatform, "Tee internal core api specification," https://globalplatform.org/specs-library/tee-internal-core-apispecification-v1-2/ 2019, accessed: 2019-12-05.

[8] GlobalPlatform, "Globalplatform current members," https: //globalplatform.org/current-members/2 2020, accessed: 2019-0115.

[9] Google, "Android keystore," 2018, https://source.android.com/security/ keystore

[10] J. Guilbon, "Attacking the arm's trustzone," https://blog.quarkslab.com/ attacking-the-arms-trustzone.html 2018, accessed: 2019-08-28.

[11] L. Harrison, H. Vijayakumar, R. Padhye, K. Sen, and M. Grace, "Partemu: Enabling dynamic analysis of real-world trustzone software using emulation," in Proceedings of the 29th USENIX Security Symposium (USENIX Security 2020) (To Appear), August 2020.

[12] Z. Hua, J. Gu, Y. Xia, H. Chen, B. Zang, and H. Guan, "vtz: Virtualizing ARM trustzone," in 26th USENIX Security Symposium (USENIX Security 17), 2017, pp. 541-556.

[13] D. Komaromy, "Unbox your phone - part i." https://medium. com/taszksec/unbox-your-phone-part-i-331bbf44c30c 2018, accessed: 2019-08-28.

[14] D. Komaromy, "Unbox your phone - part ii." https://medium.com/ taszksec/unbox-your-phone-part-ii-ae66e779b1d6 2018, accessed: 2019-08-28.

[15] D. Komaromy, "Unbox your phone - part iii." https://medium. com/taszksec/unbox-your-phone-part-iii-7436ffaff7c7 2018, accessed: 2019-08-28.

[16] L. Limited, "Open portable trusted execution environment," https: //www.op-tee.org/ 2020, accessed: 2020-01-15.

[17] S. Makkaveev, "The road to qualcomm trustzone apps fuzzing," https://research.checkpoint.com/2019/the-road-to-qualcomm-trustzoneapps-fuzzing/ 2019, accessed: 2019-11-30.

[18] N. Nethercote and J. Seward, "Valgrind: a framework for heavyweight dynamic binary instrumentation," ACM Sigplan notices, vol. 42, no. 6, pp. 89-100, 2007.

[19] NIST, “Cve-2016-8764,” https://nvd.nist.gov/vuln/detail/CVE-20168764 2017, accessed: 2019-08-28.

[20] D. Shen, "Attacking your Trusted Core," https://www.blackhat. com/docs/us-15/materials/us-15-Shen-Attacking-Your-Trusted-CoreExploiting-Trustzone-On-Android.pdf 2015, accessed: 2019-11-28.

[21] Y. Shoshitaishvili, R. Wang, C. Salls, N. Stephens, M. Polino, A. Dutcher, J. Grosen, S. Feng, C. Hauser, C. Kruegel, and G. Vigna, "SoK: (State of) The Art of War: Offensive Techniques in Binary Analysis," in IEEE Symposium on Security and Privacy, 2016.

[22] N. Stephens, "Behind the pwn of a trustzone," https: //www.slideshare.net/GeekPwnKeen/nick-stephenshow-does-someoneunlock-your-phone-with-nose 2017, accessed: 2019-08-28.

[23] N. Stephens, "Bypassing huawei's fingerprint authentication by exploiting the trustzone," https://www.youtube.com/watch?v= MdoGCXGHGnY 2018, accessed: 2019-08-28.

[24] A. Tarasikov, "Qemu teegris usermode," https://github.com/astarasikov/ qemu/tree/teegris_usermode 2019, accessed: 2019-11-30.

[25] H. Technologies, "Emui 8.0 security technical white paper," 2017, https://consumer-img.huawei.com/content/dam/huawei-cbg-site/en/ mkt/legal/privacy-policy/EMUI8.0SecurityTechnologyWhitePaper.pdf 